\title{
Enseñanza remota de emergencia ante la pandemia Covid-19 en Educación Media Superior y Educación Superior
}

\section{Emergency remote teaching in the face of the Covid-19 pandemic in Middle and Higher Education}

Samuel Alejandro Portillo Peñuelas

Instituto Tecnológico de Sonora, Sonora, México

ORCID: http://orcid.org/0000- 0002-1521-6619

Lidia Isabel Castellanos Pierra*

Centro Universitario de Sonora, Sonora, México

ORCID: https://orcid.org/0000-0002-3714-9189

\section{Oscar Ulises Reynoso González}

Centro Universitario de los Altos de la Universidad de Guadalajara, Jalisco, México

ORCID: http://orcid.org/0000-0002-0598-4665

\section{Omar Iván Gavotto Nogales}

Universidad de Sonora, Sonora, México

ORCID: http://orcid.org/0000-0001-9645-2172

Received 07-30-20 Revised 08-15-20 Accepted 09-29-20 On line 09-30-20

*Correspondence

Email: lidiaisabelcp@yahoo.es
Cite as:

Portillo, S., Castellanos, L., Reynoso, O., \& Gavotto, O. (2020). Enseñanza remota de emergencia ante la pandemia Covid-19 en Educación Media Superior y Educación Superior. Propósitos y Representaciones, 8 (SPE3), e589. Doi: http://dx.doi.org/10.20511/pyr2020.v8nSPE3.589 


\section{Resumen}

Este trabajo tiene por objetivo realizar una aproximación a las experiencias del profesorado y estudiantado de Educación Media y Superior en torno a la estrategia de enseñanza implementada durante la emergencia sanitaria por Covid-19. La muestra se integró por 44 docentes y 116 estudiantes originarios de un municipio del sur del estado de Sonora, México. Desde el punto de vista teórico se atiende a los principios de la Enseñanza Remota de Emergencia para enfrentar la crisis a través del trabajo escolar mediado por tecnología. Se diseñó un cuestionario ad hoc para explorar: a) el uso de dispositivos, conectividad y espacios alternativos de instrucción para dar continuidad a los estudios, b) la implementación de los recursos tecnológicos con base en la experiencia, dificultades y preparación, c) evaluación y apoyo recibido y d) adaptación y proyección de los aprendizajes. Los resultados evidencian el uso de laptop y teléfono inteligente como los dispositivos de mayor uso para el estudio, y el envío y recepción de información respectivamente. Además, se señala el incremento de tiempo dedicación y dificultades para la recepción-evaluación de las actividades escolares tanto en docentes como estudiantes. En cuanto al apoyo institucional la habilitación de cursos, softwares y plataformas virtuales representan las principales acciones para dar continuidad a los estudios. Destacando el esfuerzo, voluntad, manejo de tecnología, cambios de dinámica respecto al rol y la revalorización de las clases presenciales como los principales aprendizajes durante el confinamiento.

Palabras clave: Docentes; Estudiantes; Educación Media Superior; Educación Superior; Enseñanza Remota.

\section{Summary}

The objective of this work is to carry out an approach to the experiences of the teachers and students of Middle and Higher Education around the teaching strategy implemented during the health emergency by Covid-19. The sample was made up of 44 teachers and 116 students from a municipality in the south of the state of Sonora, Mexico. From a theoretical point of view, the principles of Emergency Remote Teaching are addressed to face the crisis through school work mediated by technology. An ad hoc questionnaire was designed to explore: a) the use of devices, connectivity and alternative instructional spaces to give continuity to the studies, b) the implementation of technological resources based on experience, difficulties and preparation, c) evaluation and received support and d) adaptation and projection of learning. The results show the use of laptop and smartphone as the most widely used devices for the study, and the sending and receiving of information, respectively. In addition, the increase in dedication time and difficulties for the reception-evaluation of school activities in both teachers and students is noted. Regarding institutional support, the enabling of courses, software and virtual platforms represent the main actions to give continuity to the studies. Highlighting the effort, will, technology management, changes in dynamics regarding the role and the revaluation of face-to-face classes as the main learning during confinement.

Keywords: Teachers: Students; Higher Secondary Education; Higher Education; Remote Teaching.

\section{Introducción}

Con la declaración de emergencia sanitaria que se vive a nivel mundial y la inminente suspensión de actividades escolares por el cierre de escuelas en todos los niveles educativos (Luthra, 2020) a causa del nuevo coronavirus SARS-CoV-2 conocido como Covid-19, el sector educativo enfrenta un problema más allá de la salud pública. El cual, se encuentra relacionado con los procesos de enseñanza durante la etapa de distanciamiento social, dejando en evidencia que el Sistema Educativo Nacional vive una de las peores crisis, la que estudiantes, padres de familia, profesores y otros actores, habrán de enfrentar recurriendo a estrategias educativas mediadas por tecnología como medida compensatoria del sistema presencial (Almazán, 2020; Álvarez et al., 
2020; Arce-Peralta, 2020; Arriagada, 2020; Bonilla-Guachamín, 2020; Cáceres-Piñaloza, 2020; Cóndor-Herrera, 2020; García-García, 2020; Menéndez \& Figares, 2020; Morales, 2020; Moreno-Rodríguez, 2020; Pardo \& Cobo, 2020; Pascuas-Rengifo et al., 2020; Pérez-Narváez \& Tufiño, 2020; Quintana, 2020; Ruiz, 2020; Suárez, 2020; UNESCO, 2020a; Varela et al., 2020; Vivanco-Saraguro, 2020).

La situación histórica que se vive ha obligado a los gobiernos y autoridades educativas a establecer estrategias para garantizar la continuidad de los estudios (Casal \& Fernández, 2020; Ruíz, 2020), de la misma forma que el profesorado de los diversos subsistemas de bachillerato y las universidades, han tenido que rediseñar y adaptar los contenidos planeados hacia la transición virtual. En este sentido, la estrategia que se ha generalizado por ajustarse a las necesidades de dichos niveles, ha sido el trabajo mediado por tecnología digital, el cual permite añadir a las necesidades académicas, redes de apoyo conformadas por comunidades escolares que permitan enfrentar la crisis, concibiendo "las experiencias de aprendizaje remoto como un aspecto central en el proceso integral de formación” (Pardo \& Cobo, 2020, p.8) del estudiantado.

Pasar de experiencias de formación presenciales a otras exclusivas en plataformas digitales ha puesto de manifiesto que el aislamiento social trae como consecuencia desigualdad y generación de brechas entre sectores de la sociedad (Arriagada, 2020; Bocchio, 2020; Casal \& Fernández, 2020; Cifuentes-Faura, 2020; Pardo \& Cobo, 2020). Hay quienes muestran resistencia a los cambios y otros que carecen de los recursos necesarios para hacer frente a ellas. Si a esto, se le suma la falta de acceso a tecnología, aumentan las barreras (Álvarez et al., 2020), provocando a simple vista en el ámbito social dos grandes extremos, familias que viven en condiciones precarias y otras que cuentan con todos los recursos necesarios para enfrentar el problema. Siendo el mismo resultado si realizamos dicho ejercicio volteando la mirada hacia los centros escolares. Por un lado, planteles del sector público con la condición generalizable de ausencia de servicios básicos y materiales de estudio, y por el otro, planteles del sector privado que responden al alumnado con trabajo diario basado en estrategias que garantizan el uso de tecnologías y plataformas educativas.

En este escenario, una parte del estudiantado y profesorado no podrá adaptarse, "ya sea por falta de recursos o facilidades en su lugar de confinamiento" (Menéndez \& Figares, 2020, p.2), por falta de infraestructura del plantel y/o nivel de desarrollo digital necesario para suplir los programas con educación a distancia (Pardo \& Cobo, 2020; Ruíz, 2020), entre otros factores asociados a situaciones familiares y de salud pública, con consecuencias más graves en los sectores más desfavorecidos (Bocchio, 2020), dando apertura a la brecha virtual (Almazán, 2020; Luthra, 2020).

Para mitigar las distancias en cuanto al acceso de contenidos educativos por parte del estudiantado en el contexto mexicano, se han implementado sesiones de clase a través de canales de televisión abierta para minimizar el rezago, la deserción y los riesgos de exclusión (Almazán, 2020) en Educación Básica. Garantizando con ello, el derecho a la educación (Ruíz, 2020). El programa nacional denominado Aprende en Casa, es la iniciativa que la Secretaría de Educación Pública (SEP) diseñó e implementó de manera emergente para la educación obligatoria en México (preescolar, primaria, secundaria y nivel medio superior) (Mérida \& Acuña, 2020).

A medida que la cuarentena ha ido ampliándose, se ha comenzado a hablar indistintamente de "educación online, educación remota, educación virtual y educación digital" (Bustamante, 2020, p.2) La situación inédita de emergencia ha obligado a que las escuelas recurran a distintas estrategias para dar continuidad a los periodos lectivos (Álvarez et al., 2020) compartiendo algunas en común e implementando otras a nivel particular y específico de las necesidades del contexto y tipo de población escolar a la que atienden. Ello, representa el caso de las Universidades (Educación Superior) quienes al igual que los planteles de Educación Básica, aunque con mayor estructura, "no estaban preparados para una crisis como la que se vive" (Bus- 
tamante, 2020; Casal \& Fernández, 2020) por lo que de igual forma, han recurrido a todas las herramientas tecnológicas disponibles para enfrentar la contingencia y atender la demanda educativa.

En este sentido, lo que se planteó en un inicio como una estrategia temporal se ha volcado permanente, lo que ha obligado a hablar de Educación Remota de Emergencia (Bustamante, 2020). Dicho término se propuso por Hodges et al., (2020) para diferenciar entre el tipo de instrucción que se da en condiciones normales (Enseñanza tradicional/cotidiana) y otro que se imparte en circunstancias apremiantes (Enseñanza de emergencia/extraordinaria), siendo el tiempo, las prisas y el uso mínimo de recursos para realizar tareas de enseñanza y aprendizaje, las condiciones particulares del término. Alude al contexto universitario y a la necesidad que tiene dicho espacio de continuar generando conocimiento desde fuera de la universidad (Tabla 1). En este sentido, el contexto de emergencia se ha caracterizado por "limitaciones tanto en el control de la enseñanza, como por las restricciones de las interacciones entre profesores y alumnos, y alumnos entre sí" (Álvarez et al., 2020, p.28)

Tabla 1.

Modelo de evaluación de Enseñanza Remota de Emergencia

\begin{tabular}{|c|c|c|c|}
\hline $\begin{array}{c}\text { Evaluaciones de con- } \\
\text { texto }\end{array}$ & Evaluación de entrada & $\begin{array}{c}\text { Evaluaciones de } \\
\text { proceso }\end{array}$ & $\begin{array}{c}\text { Evaluaciones de } \\
\text { producto }\end{array}$ \\
\hline $\begin{array}{l}\text { - Identificación de re- } \\
\text { cursos internos y ex- } \\
\text { ternos necesarios para } \\
\text { la transición. } \\
\text { - Interacción entre per- } \\
\text { sonal, estudiantes, } \\
\text { familias, institución y } \\
\text { autoridades locales. }\end{array}$ & $\begin{array}{l}\text { - Infraestructura tecnoló- } \\
\text { gica. } \\
\text { - Capacidad del personal } \\
\text { para enfrentar la crisis. } \\
\text { - Uso de espacios alterna- } \\
\text { tivos de instrucción y } \\
\text { aprendizaje }\end{array}$ & $\begin{array}{l}\text { - Dificultades, obs- } \\
\text { táculos y limitan- } \\
\text { tes de estudiantes, } \\
\text { personal docente y } \\
\text { centros escolares }\end{array}$ & $\begin{array}{l}\text { - Aprendizajes } \\
\text { obtenidos en } \\
\text { contextos de } \\
\text { emergencia y } \\
\text { satisfacción } \\
\text { con los resulta- } \\
\text { dos } \\
\text { - Retos y desa- } \\
\text { fíos. }\end{array}$ \\
\hline
\end{tabular}

Nota: Elaboración propia con base en Hodges, et al., (2020). The Difference Between Emergency Remote Teaching and Online Learning. Recuperado de https://bit.ly/3cng48F

Con base en lo anterior, el contexto de emergencia, más allá de priorizar la evaluación como parte final de la estrategia, debe profundizar en todo lo necesario para adecuarse al contexto en un corto periodo de tiempo (Hodges et al., 2020). Ello, advierte diversos problemas al momento de su implementación. En el caso de Educación Media Superior, lo que se advierte, es el acceso a una programación que se encuentra limitada en horarios y organizada en contenidos estandarizados. Es decir, quien sólo dispone de un canal de acceso a los contenidos (en este caso televisión abierta) debe adaptarse a la programación establecida, contextualizando que a nivel nacional existen diferencias de horarios por regiones geográficas dentro del territorio nacional. Por otra parte, las sesiones clase, abordan contenidos estandarizados orientados al alumnado a nivel general, siendo sólo apto para aquellos que se encuentran en condiciones académicas regulares. Ello, evidencia que la programación no contempla al estudiantado que presenta necesidades específicas de apoyo. Ahí, es donde juega un papel determinante el profesorado, quien de manera complementaria envía actividades acordes al nivel académico del alumnado con condiciones particulares para el aprendizaje.

Lo anterior, ha propiciado que se realicen esfuerzos en cada sector, zona escolar y planteles educativos por plantear estrategias de enseñanza remota. Ello, sin que existan criterios de calidad de un plantel educativo a otro (Arriagada, 2020). En este sentido, dependiendo de la escuela en que se encuentre inscrito el estudiante recibirá sus clases de modo remoto y síncrono. $\mathrm{O}$ bien, en otros casos el profesorado preparará material y videos para que el estudiantado los 
revise de manera asíncrona (Lederman, 2020). También estará el estudiantado que recibirá instrucciones y contenidos a través de dispositivos móviles en redes sociales, o bien escucharán la radio y/o visualizarán la televisión (Bustamante, 2020). Ello, de entrada, denota contrastes entre quienes realizan sus estudios en tiempo de pandemia con recursos limitados y aquellos que presentan mejores condiciones para realizar el trabajo en casa. Mientras algunos estudiantes no tienen acceso a señal de internet por dificultades geográficas (barrera de accesibilidad tecnológica), otros si la tienen, pero no pueden pagarla (barrera económica) (Arriagada, 2020) o no cuentan con una computadora para uso personal (barrera material) (Quintana, 2020) entre otras limitantes.

Si a ello, agregamos el estudiantado que presenta condiciones de Necesidades Educativas Especiales (NEE) o de discapacidad, y que requieren atención diferenciada, la escuela en su transición hacia la virtualidad enfrenta un gran reto en cuanto a realizar a la brevedad las adecuaciones pertinentes para practicar estrategias de inclusión que se dicen promover en entornos escolares presenciales (Jiménez, 2020; Moreno-Rodríguez, 2020; Rodríguez del Rincón, 2020).

Otro problema generado al implementarse un sistema de enseñanza remota "es la invisibilización de las diferentes condiciones de acceso a los recursos digitales" (Álvarez et al., 2020, p.34) Ello, es un indicativo que aunque el modelo de Enseñanza Remota de Emergencia debe encontrar formas de satisfacer la necesidad de continuidad educativa mientras se vive en un entorno de crisis (Hodges et al., 2020), sigue una lógica individual (Ruiz, 2020; Álvarez et al., 2020) donde "el principal elemento mediador tecnológico es el celular, la tableta o cualquier dispositivo electrónico móvil" (Pascuas-Rengifo et al., 2020, p.99). Ello, debido al uso que se ha venido dando a aplicaciones como Zoom, Google Meet, Teams y otros tantos sistemas de videoconferencia (Moreno-Rodríguez, 2020), además de aplicaciones de mensajería instantánea como WhatsApp y Telegram, siendo esta últimas las más utilizadas para el envío de actividades curriculares (Bocchio, 2020). Esta realidad, demuestra que "las universidades están promoviendo la adopción y el uso intensivo de diferentes plataformas de tecnología digital (como Google, Facebook, Amazon AWS, YouTube, entre otras) cuyos servicios se ofrecen de manera gratuita para servir y empoderar a estudiantes, académicos y administradores" (Pardo \& Cobo, 2020, p.63).

Con base a lo anteriormente expuesto, se puede afirmar que la educación mediada por tecnología implementada de manera emergente incrementa la desigualdad educativa y evidencia las brechas referentes a infraestructura, conectividad y un mundo de posibilidades de usos formativos entre el profesorado y estudiantado (Ruiz, 2020) que tiene las condiciones y los que no. Mismas diferencias se visualizan en cuanto a la organización del profesorado en cuanto a las solicitudes de actividades escolares, la preocupación por el avance en los contenidos y la organización de los tiempos para el cumplimiento de los objetivos considerando el contexto geográfico en el que se ubican los centros escolares (Muñoz \& Lluch, 2020). Lo anterior, evidencia que desde la virtualidad se han planteado diversas lógicas de trabajo en tiempos de pandemia, las cuales varían desde el cumplimiento de horarios, entrega de actividades, adaptación de contenidos, modificación del currículo, el paso de las tareas académicas a las tareas domésticas que permitan aprender otro tipo de habilidades, el desarrollo de habilidades emocionales y prácticas de comunicación y socialización para la integración familiar, entre otras.

En este contexto de emergencia, el profesorado en los diferentes niveles educativos ha tenido que reinventarse de la noche a la mañana, integrando forzosamente nuevas herramientas de trabajo, más allá del papel, las evidencias y otros instrumentos formativos (Arriagada, 2020) para hacer frente a las nuevas demandas que, sumadas a las ya existentes, se adhieren en el contexto de la virtualidad (Jiménez, 2020; Menéndez \& Figares, 2020). Tiempo de planeación y preparación de clases, revisión de recursos, adaptación de contenidos, así como el diseño, envío, recepción y retroalimentación de actividades por estudiante, son algunos de los desafíos a los que se han tenido que adaptar (Ruíz, 2020). En este sentido, la experiencia de trabajar en am- 
bientes mediados por tecnología, ha generado una acelerada digitalización de contenidos educativos (Almazán, 2020) y posibilitado la adopción del aprendizaje en línea (Lederman, 2020).

Resulta evidente la necesidad de usar las tecnologías digitales para asegurar que la enseñanza remota en sus distintos escenarios no se interrumpa (Pardo \& Cobo, 2020) (Tabla 2), siendo los dispositivos móviles una importante herramienta en esta estrategia (Pascuas-Rengifo et al., 2020) para el trabajo en línea. No obstante, la condición de emergencia pública no sólo requiere del aspecto tecnológico, sino también del organizativo, ya que exige cambios vertiginosos en cuanto los procesos de planeación de las clases, los cuales pasan de un periodo de meses en condiciones anormales a un plazo de semanas o días en contextos de emergencia (Hodges et al., 2020). En este sentido, se enuncia otro gran problema, relacionado a la disminución de la calidad de los cursos impartidos, específicamente en cuanto a la distinción de los materiales de aprendizaje que deben ser aprendidos y aquellos que deben ser pospuestos durante el tiempo que dure el problema, conocido como disolución del currículum escolar (Álvarez et al., 2020; Casal \& Fernández, 2020; Morales, 2020). Aunado a ello, las nuevas limitantes generadas a la hora de su ejecución debido a los tiempos de planificación (Álvarez et al., 2020). Será "la velocidad impuesta para disponer de dichos recursos, la distancia, la virtualidad y el aislamiento lo que restrinja el papel creativo de los docentes en relación con el tiempo para la planificación" (Ibid, 2020, p.38).

En este sentido, al igual que el profesorado, tanto el alumnado, como sus familias, se han visto en la necesidad de adaptarse a los requerimientos, herramientas e instrumentos mínimos para continuar con el temario y las tareas escolares desde casa (Muñoz \& Lluch, 2020), de tal manera que el apoyo familiar en los hogares que se encuentran en confinamiento resulta determinante para el éxito de la estrategia de aprender en casa (Cifuentes-Faura, 2020).

Tabla 2.

Escenarios de la Docencia Remota de Emergencia (DRE)

\begin{tabular}{|c|c|c|c|c|}
\hline & Metas instruccionales & Método & Materiales & $\begin{array}{l}\text { Evalua- } \\
\text { ción }\end{array}$ \\
\hline $\begin{array}{l}\text { Remoto } \\
\text { Urgente No } \\
\text { Adaptado }\end{array}$ & $\begin{array}{l}\text { Se aprenden los con- } \\
\text { tenidos previstos para } \\
\text { cumplir en la crisis y } \\
\text { aprobar la asignatura } \\
\text { con una calificación. } \\
\text { El nivel de exigencia } \\
\text { es más bajo ante una } \\
\text { mayor tolerancia y } \\
\text { empatía por el confi- } \\
\text { namiento. Los estu- } \\
\text { diantes tendrán que } \\
\text { dedicar más tiempo a } \\
\text { la comprensión del } \\
\text { objeto de estudio. }\end{array}$ & $\begin{array}{l}\text { Se adapta poco el método } \\
\text { tradicional y se improvi- } \\
\text { san las decisiones. Los } \\
\text { docentes son el centro de } \\
\text { la clase y los grandes di- } \\
\text { vulgadores de contenidos. } \\
\text { Las sesiones de videocon- } \\
\text { ferencias son largas y se } \\
\text { mantiene la idea de un } \\
\text { trabajo final para la cali- } \\
\text { ficación. El uso de tecno- } \\
\text { logía se basa en las nece- } \\
\text { sidades de adaptación } \\
\text { imperando lo analógico } \\
\text { en el "nuevo" contexto } \\
\text { remoto. }\end{array}$ & $\begin{array}{l}\text { Los mis- } \\
\text { mos con- } \\
\text { tenidos } \\
\text { previstos } \\
\text { con mate- } \\
\text { rial esca- } \\
\text { neado para } \\
\text { distribu- } \\
\text { ción vía } \\
\text { electróni- } \\
\text { ca. }\end{array}$ & $\begin{array}{l}\text { La mis- } \\
\text { ma cali- } \\
\text { ficación } \\
\text { prevista, } \\
\text { pero con } \\
\text { bajo ni- } \\
\text { vel de } \\
\text { exigen- } \\
\text { cia. }\end{array}$ \\
\hline
\end{tabular}




\begin{tabular}{|c|c|c|c|c|}
\hline $\begin{array}{l}\text { Remoto } \\
\text { Urgente } \\
\text { Adaptado }\end{array}$ & $\begin{array}{l}\text { Se aprenden los con- } \\
\text { tenidos previstos para } \\
\text { cumplir en la crisis y } \\
\text { aprobar la asignatura } \\
\text { con una calificación. } \\
\text { Existe mayor toleran- } \\
\text { cia y empatía por la } \\
\text { situación de confina- } \\
\text { miento: nivel de exi- } \\
\text { gencia y expectativas } \\
\text { más bajo que en la } \\
\text { asignatura presencial. }\end{array}$ & $\begin{array}{l}\text { Existe un menor margen } \\
\text { de improvisación por par- } \\
\text { te de los docentes aunque } \\
\text { el proceso de aprendizaje } \\
\text { gira en torno a éstos, ya } \\
\text { que son el participante } \\
\text { más inteligente del aula. } \\
\text { Las sesiones de videocon- } \\
\text { ferencias son más breves } \\
\text { con mentorías individua- } \\
\text { les y entrega de trabajos } \\
\text { finales. El esfuerzo del } \\
\text { alumnado se basa en ex- } \\
\text { periencias previas y adap- } \\
\text { tación a sus necesidades } \\
\text { curriculares. }\end{array}$ & $\begin{array}{l}\text { Se realiza } \\
\text { un ajuste y } \\
\text { curación } \\
\text { de conte- } \\
\text { nidos. Or- } \\
\text { ganizados } \\
\text { de manera } \\
\text { cronológi- } \\
\text { ca o en } \\
\text { listado de } \\
\text { bibliogra- } \\
\text { fía sugeri- } \\
\text { da por el } \\
\text { docente. }\end{array}$ & $\begin{array}{l}\text { Se aña- } \\
\text { den di- } \\
\text { námicas } \\
\text { de auto- } \\
\text { evalua- } \\
\text { ción y } \\
\text { evalua- } \\
\text { ción por } \\
\text { pares } \\
\text { (dado el } \\
\text { menor } \\
\text { control } \\
\text { docente } \\
\text { de los } \\
\text { equipos) }\end{array}$ \\
\hline $\begin{array}{l}\text { Remoto } \\
\text { Aspiracio- } \\
\text { nal }\end{array}$ & $\begin{array}{l}\text { Existe una secuencia } \\
\text { de aprendizaje bien } \\
\text { clara. Las metas se } \\
\text { dirigen al aprendizaje } \\
\text { basado en la resolu- } \\
\text { ción de retos y el do- } \\
\text { cente colabora con } \\
\text { otros docentes del } \\
\text { mismo año lectivo } \\
\text { para producir juntos } \\
\text { cátedras integradoras. }\end{array}$ & $\begin{array}{l}\text { El docente tiende a hacer- } \\
\text { se invisible a través del } \\
\text { diseño de experiencias de } \\
\text { aprendizaje que pone al } \\
\text { estudiante al centro, colo- } \\
\text { cándolo al grupo como el } \\
\text { participante más inteli- } \\
\text { gente del aula. Existe cla- } \\
\text { ridad en los tiempos de } \\
\text { trabajo, se reduce la im- } \\
\text { provisación y se gestio- } \\
\text { nan grupos de trabajo en } \\
\text { modalidad síncrona y } \\
\text { asíncrona. Las sesiones } \\
\text { son breves (No más de } 30 \\
\text { minutos). Se añade la ges- } \\
\text { tión de la seguridad del } \\
\text { estudiante en cuanto a su } \\
\text { privacidad en el manejo } \\
\text { de cuentas de acceso. }\end{array}$ & $\begin{array}{l}\text { Se realiza } \\
\text { una mayor } \\
\text { curación } \\
\text { de conte- } \\
\text { nidos, dis- } \\
\text { tinguiendo } \\
\text { los recur- } \\
\text { sos pro- } \\
\text { pios y ex- } \\
\text { ternos. } \\
\text { Además } \\
\text { de poder } \\
\text { integrar } \\
\text { otros con- } \\
\text { siderados } \\
\text { de calidad } \\
\text { por los } \\
\text { estudian- } \\
\text { tes. Existe } \\
\text { mayor } \\
\text { apertura a } \\
\text { formatos } \\
\text { no textua- } \\
\text { les. }\end{array}$ & $\begin{array}{l}\text { Se inte- } \\
\text { gran di- } \\
\text { námicas } \\
\text { gamifi- } \\
\text { cadas y } \\
\text { otros } \\
\text { formatos } \\
\text { de } \\
\text { autoeva- } \\
\text { luación y } \\
\text { evalua- } \\
\text { ción por } \\
\text { pares (la } \\
\text { evalua- } \\
\text { ción es } \\
\text { un proce- } \\
\text { so más } \\
\text { reticu- } \\
\text { lar). }\end{array}$ \\
\hline
\end{tabular}

Nota: Elaboración propia con base en Pardo y Cobo (2020). Expandir la universidad más allá de la enseñanza remota de emergencia: Ideas hacia un modelo híbrido postpandemia. Outliers School. Barcelona.

En el caso de experiencias internacionales con respecto a la estrategia educativa implementada en situaciones de emergencia Hodges et al., (2020) mencionan el caso de Afganistán donde es el caso de algunas escuelas y Universidades que han tenido que responder al cierre por contextos diversos, en los cuales los modelos de aprendizaje móvil, radio y otras estrategias de aprendizaje combinado han representado soluciones factibles. En el caso de América Latina destaca el caso de Perú con el programa Aprendo en Casa y la programación que se ha hecho considerando que en casi todas las casas del país hay un teléfono móvil, y es a través de este aparato que la mayoría de peruanos se conecta a internet (Bustamante, 2020). En el caso de Colombia, para hacer frente a la emergencia provocada por la pandemia de Covid-19 el estado Co- 
lombiano permitió el acceso gratuito a internet de las líneas de telefonía celular prepagadas, con la intencionalidad de garantizar el desarrollo de las actividades escolares (Pascuas-Rengifo et al., 2020). En Chile, para minimizar el rezago por el cierre de centros educativos, se han mantenido las aulas abiertas desde la distancia mediante internet (Quintana, 2020) realizando esfuerzos para que los más desfavorecidos puedan disponer de ordenadores portátiles, tabletas informáticas o puntos de conexión para móviles (Luthra, 2020).

En México, se ha identificado el programa Aprende en Casa en Educación Básica y Media Superior, así como el de Educación Remota de Emergencia en las universidades. Además, con respecto al desarrollo de análisis en torno a la problemática educativa en tiempos de pandemia sólo se han identificado dos trabajos relacionados al impacto de la Covid-19 en el ámbito educativo, ambos relacionados a las medidas prioritarias y de acción primarias implementadas. Relacionadas estas con la suspensión de actividades escolares ante la emergencia, los programas de educación implementados y las estrategias de trabajo extraescolares que se realizaron en Educación Media Superior y Educación Superior vía remota, ambos considerados como medidas paliativas emergentes en tiempos de confinamiento (Cervantes \& Gutiérrez, 2020; Mérida \& Acuña, 2020).

Con base a lo anterior, el artículo responde a los siguientes cuestionamientos ¿Cuál ha sido la experiencia del profesorado y estudiantado en el contexto de Enseñanza Remota de Emergencia de Educación Media y Educación Superior en cuanto al uso de espacios alternativos de instrucción para dar continuidad a los estudios de bachillerato y universitarios? ¿Cómo se ha organizado la comunidad educativa para mantener comunicación, relaciones de apoyo y enfrentar las múltiples dificultades durante el desarrollo de los proyectos de aprendizaje? ¿Cómo han tenido que cambiar las dinámicas en cuanto al rol, uso de dispositivos, espacios y tiempos de trabajo en el profesorado y estudiantado que trabaja y estudia vía remota en tiempos de emergencia desde casa? ¿Cuáles han sido los mayores aprendizajes del profesorado y estudiantado durante el confinamiento? En este sentido, se plantea como objetivo analizar la experiencia del profesorado y estudiantado de bachillerato y universidad que ha participado en la estrategia de Enseñanza Remota de Emergencia implementada en México, durante la pandemia de Covid-19 delimitando cuatro grandes categorías de exploración: Dispositivos, conectividad y espacios alternativos de instrucción; experiencia previa, dificultades y preparación; evaluación y apoyo recibido; transformación y proyección de los aprendizajes.

\section{Método}

\section{Diseño}

De acuerdo con la clasificación de Hernández y Mendoza (2018), el presente estudio se desarrolló bajo el enfoque cuantitativo de investigación, con un diseño transversal, observacional (no experimental) y de alcance exploratorio.

\section{Participantes}

A través de un muestreo no probabilístico de tipo incidental, fueron seleccionados 44 docentes y 116 estudiantes de educación media y superior originarios de un municipio del sur del Estado de Sonora, México. Los participantes pertenecen predominantemente a un contexto urbano $(82.5 \%)$. Con respecto a los docentes, el $56.8 \%$ eran hombres y $43.2 \%$ mujeres, con promedio de edad de $39.3(D E=7.57)$, el $27.3 \%$ se desempeña en bachillerato y el $72.7 \%$ en licenciatura en el área de Educación; la mayoría cuenta con más de 5 años de experiencia (86.4\%). Por su parte, en la muestra de estudiantes, el $16.4 \%$ eran hombres y $83.6 \%$ mujeres, con promedio de edad de $21.7(D E=4.20)$, el $21.6 \%$ del estudiantado se encuentra cursando el bachillerato y el 78.4\% una licenciatura en el área de Ciencias de la Educación. 


\section{Instrumento}

Para la recolección de información se diseñó un cuestionario ad hoc para conocer la experiencia del profesorado y estudiantado después de dos meses de haberse implementado la enseñanza remota de emergencia por la pandemia de Covid-19. Las preguntas incluidas se desarrollaron bajo los siguientes tópicos:

- Dispositivos, conectividad y espacios alternativos de instrucción: Aborda el acceso a internet y la disposición de dispositivos para seguir trabajando en la modalidad en línea

- Experiencia previa y dificultades: Incluye el acercamiento previo a la modalidad de educación a distancia y la enunciación de obstáculos y dificultades en el proceso

- Evaluación y apoyo recibido: Se hace una valoración sobre el soporte recibido por docentes, padres e instituciones y sobre la experiencia de enseñanza remota de emergencia

- Transformación y proyección de los aprendizajes: Integra una reflexión sobre la forma en que los roles de docente y alumno fueron transformados en la experiencia de enseñanza remota y un pronóstico sobre el propio proceso de enseñanza aprendizaje

\section{Procedimiento, análisis de datos y consideraciones éticas}

Primeramente, se construyó el cuestionario Estrategia Remota de Emergencia en Educación Media y Superior con base en cuatro grandes categorías de análisis: Dispositivos, conectividad y espacios alternativos de instrucción; experiencia previa y dificultades; evaluación y apoyo recibido; transformación y proyección de los aprendizajes. Mismo que se trasladó a la herramienta formularios de Google para facilitar la recabada de datos atendiendo a los protocolos de sana distancia, además de facilitar el manejo, difusión y el medio de respuestas a través de dispositivos móviles.

Para la fase de recogida de datos, se solicitó la aprobación a dos centros de Bachillerato y dos Universidades ubicadas en un municipio del sur del estado de Sonora. Los centros apoyaron con la difusión y aplicación del cuestionario a través del envío de la liga que re-direcciona al instrumento. El proceso de recolección se realizó durante el periodo de un mes al cierre del ciclo escolar 2019-2020. La condición para dar respuesta fue cumplir con el rol de docente o estudiante de Educación Media o Superior, previa aceptación voluntaria de participación y consentimiento informado de uso exclusivo de datos para análisis estadísticos y presentación de información con fines académicos, garantizando los principios de participación anónima, sin alguna compensación a cambio. El proceso de respuesta estimado para contestar el cuestionario fue de 20 minutos.

Al ser un estudio de alcance exploratorio, los resultados se presentan a través de estadística descriptiva, utilizando mayormente frecuencias y porcentajes. Además, de utilizar medidas de tendencia central o dispersión en los casos que el formato de respuesta del cuestionario da oportunidad. En algunos casos se precisan comparaciones entre los niveles de estudio (bachillerato y universidad) para aspectos en los que resulta necesario explorar y profundizar, sin que se pretenda establecer diferencias estadísticamente significativas entre grupos. El software utilizado para el análisis de los datos fue SPSS V. 21.

\section{Resultados}

Para dar orden a la presentación de resultados, se utilizaron las categorías de análisis como guías, mostrando las respuestas del profesorado y alumnado en cada una de ellas. 


\section{Dispositivos, conectividad y espacios alternativos de instrucción}

Ambos actores reportaron hacer uso de distintos dispositivos para continuar con sus clases en formato a distancia, siendo la computadora o laptop el más utilizado. Sin embargo, mientras que el $95.5 \%$ del profesorado la mencionó como el dispositivo principal, el porcentaje del estudiantado fue mucho menor (67.2\%), ya que el teléfono inteligente fungió una función especial para algunos (31.9\%). Incluso, con respecto a la propiedad de estos dispositivos, en un $97.7 \%$ de los casos, los equipos eran propiedad del profesorado, mientras que para el estudiantado fue de $84.5 \%$. El porcentaje restante utilizaba dispositivos prestados por personas cercanas a ellos o eran propiedad de las instituciones donde estudiaban. Aunado a ello, la conectividad a internet también presentó algunas diferencias. Mientras que el $86.4 \%$ del profesorado tiene internet de banda ancha, sólo $25.0 \%$ del estudiantado reportó contar con este servicio. El porcentaje restante accedía a internet por servicios limitados (50.9\%) o los datos celulares (12.9\%). Un 11.2\% confirmó no tener ninguna forma de conexión a internet en el hogar (Tabla 3 ).

Tabla 3.

Dispositivos y conectividad por actor y nivel de estudios

\begin{tabular}{|l|c|c|c|c|}
\hline & \multicolumn{2}{|c|}{ Docentes } & \multicolumn{2}{c|}{ Estudiantes } \\
\hline Indicador & $\begin{array}{c}\text { Bachille- } \\
\text { rato }\end{array}$ & $\begin{array}{c}\text { Licenciatu- } \\
\text { ra }\end{array}$ & $\begin{array}{c}\text { Bachille- } \\
\text { rato }\end{array}$ & $\begin{array}{c}\text { Licencia- } \\
\text { tura }\end{array}$ \\
\hline Computadora como dispositivo principal & $91.7 \%$ & $96.9 \%$ & $56.0 \%$ & $70.3 \%$ \\
\hline $\begin{array}{l}\text { Teléfono inteligente como dispositivo prin- } \\
\text { cipal }\end{array}$ & $8.3 \%$ & $3.1 \%$ & $40.0 \%$ & $29.7 \%$ \\
\hline Acceso a internet de banda ancha & $83.3 \%$ & $87.5 \%$ & $24.0 \%$ & $25.3 \%$ \\
\hline Propiedad personal de los dispositivos & $100 \%$ & $96.9 \%$ & $84.0 \%$ & $84.6 \%$ \\
\hline $\begin{array}{l}\text { Whatsapp como medio de comunicación } \\
\text { predilecto }\end{array}$ & $91.7 \%$ & $90.6 \%$ & $48.0 \%$ & $80.2 \%$ \\
\hline
\end{tabular}

Nota: Elaboración propia con base en los resultados del SPSS V. 21

Con respecto a la comunicación entre estos actores, se utilizó una combinación de plataformas, como el correo electrónico, las redes sociales, entre otras, aunque los grupos de WhatsApp fueron el medio más popular de interacción, ya que fue utilizado como medio predilecto por el $90.1 \%$ del profesorado y el $73.3 \%$ del estudiantado. Calificando como buena la comunicación (81.9\%) entre el estudiantado y el profesorado.

En esta misma categoría se cuestionó sobre las acciones emprendidas por las instituciones para asegurar la continuidad de las clases. El estudiantado citó tres medidas adoptadas principalmente (generalmente usadas en conjunto): La habilitación de plataformas para continuar las clases en línea (73.3\%) el envío de actividades escolares por correo electrónico u otro medio (72.4\%), y el programa “Aprende en Casa" (22.4\%).

Por último, se cuestionó sobre los recursos usados para facilitar el proceso de enseñanza aprendizaje durante la enseñanza remota de emergencia. En este caso, el alumnado reportó la forma en que el profesorado empleó una combinación de recursos, en la que se destacaron el uso de libros y materiales digitalizados $(79.3 \%)$ y los vídeos $(75.9 \%)$. En menor medida, se reportó el uso de podcast $(7.0 \%)$ y blogs $(7.0 \%)$. 


\section{Experiencia previa y dificultades}

Reconociendo la importancia de los acercamientos previos a la educación a distancia, se cuestionó tanto al profesorado como al alumnado sobre su experiencia en tal modalidad (Tabla 4). Los resultados exhibieron una gran discrepancia entre los actores, mientras que el $65.9 \%$ de todo el profesorado aseguró tener antecedentes con el trabajo a distancia, solo el $25.9 \%$ del alumnado pudo confirmar haber tenido un acercamiento a dicha modalidad de estudio. De hecho, un porcentaje del profesorado advirtió no estar preparado para el cambio en la modalidad, ya que en promedio sólo el $63.7 \%$ [41.6\% para docentes de bachillerato y $71.9 \%$ de licenciatura] consideró tener las herramientas suficientes para ello. Por otro lado, al hablar de las dificultades que representó el cambio de modalidad, el profesorado destacó la recepción y evaluación de las actividades escolares de los estudiantes (47.7\%), la explicación de contenidos y aclaración de dudas por medio de los canales disponibles (34.1\%) y la planeación y adaptación de los contenidos curriculares a la modalidad a distancia (11.4\%) como los elementos más complicados para ellos. Esto conllevó una mayor carga de trabajo, ya que el 88.6\% confirmó que el tiempo dedicado a la labor docente se incrementó significativamente y, aunque el esfuerzo fue mayor, un $11.4 \%$ mencionó que su remuneración salarial se vio afectada negativamente.

Tabla 4.

Experiencia previa y tiempo dedicación por actor y nivel de estudios

\begin{tabular}{|l|c|c|c|c|}
\hline & \multicolumn{2}{|c|}{ Docentes } & \multicolumn{2}{c|}{ Estudiantes } \\
\hline Indicador & $\begin{array}{c}\text { Bachillera- } \\
\text { to }\end{array}$ & $\begin{array}{c}\text { Licenciatu- } \\
\text { ra }\end{array}$ & $\begin{array}{c}\text { Bachillera- } \\
\text { to }\end{array}$ & Licenciatura \\
\hline $\begin{array}{l}\text { Experiencia previa en educación a dis- } \\
\text { tancia }\end{array}$ & $66.7 \%$ & $65.6 \%$ & $4.0 \%$ & $31.9 \%$ \\
\hline $\begin{array}{l}\text { Tiempo dedicación trabajo/estudio } \\
\text { (Al día) }\end{array}$ & $\begin{array}{c}\text { Más de 4 } \\
\text { horas }\end{array}$ & $\begin{array}{c}\text { Más de 4 } \\
\text { horas }\end{array}$ & $\begin{array}{c}\text { De 2 a 4 } \\
\text { horas }\end{array}$ & $\begin{array}{c}\text { Más de } 4 \\
\text { horas }\end{array}$ \\
\hline
\end{tabular}

Nota: Elaboración propia con base en los resultados del SPSS V. 21

Aunado a lo anterior, el profesorado identificó un conjunto de obstáculos para que el estudiantado atendiera las actividades e indicaciones que le eran solicitadas. Cada uno de los respondientes pudo enunciar uno o más obstáculos identificados de acuerdo con su propia experiencia. En las respuestas se destacó la disposición de recursos tecnológicos (equipos o conexión a internet) (79.5\%) [66.6\% para el profesorado de bachillerato y $84.3 \%$ de licenciatura], la falta de compromiso y responsabilidad con las tareas escolares solicitadas (59.1\%) [75.0\% para el profesorado de bachillerato y $53.1 \%$ de licenciatura], la aparición de otro tipo de problemáticas familiares a partir de la pandemia (43.2\%) [33.3\% para el profesorado de bachillerato y $46.7 \%$ de licenciatura] y a un desarrollo deficiente de habilidades para el estudio independiente (43.2\%) [16.6\% para docentes de bachillerato y $53.1 \%$ de licenciatura].

\section{Evaluación y apoyo recibido}

En esta categoría se realizó una valoración sobre el apoyo recibido de parte de los padres de familia y las instituciones donde se estudia/labora. En primera instancia, al hablar de la implicación de la familia en las tareas escolares, el 52.3\% del profesorado lo consideró adecuado, el $29.5 \%$ como regular o intermitente y un $18.2 \%$ lo calificó como pésimo o inexistente. En el caso del apoyo institucional, el $68.1 \%$ lo consideró bueno o excelente, el $18.2 \%$ como regular y un $13.7 \%$ como malo o pésimo. Al abordar específicamente las gestiones institucionales efectuadas para apoyarlos en el tránsito hacia la enseñanza remota, el profesorado resaltó una o más acciones ejercidas por sus respectivas entidades, en las que se destacaron el ofrecimiento de cursos y 
asesorías al personal educativo (72.7\%), la habilitación de softwares y plataformas virtuales para la continuidad de las clases (63.6\%), la elaboración de guías para orientar el trabajo del colectivo docente durante la emergencia sanitaria (36.4\%), la gestión de materiales digitalizados para los estudiantes (29.5\%), y, en menor medida, se ofrecieron dispositivos tecnológicos para apoyar la enseñanza remota a quienes lo requerían (11.4\%). No obstante, un $13.6 \%$ reportó no haber recibido ningún tipo de apoyo por parte de su institución.

El estudiantado por su parte, confirmó tener un gran soporte familiar, ya que el $81.0 \%$ de los respondientes aseguró haber contado con el apoyo de sus padres o tutores [88.0\% para el estudiantado de bachillerato y $79.1 \%$ para el de licenciatura]. En relación al ámbito escolar, el estudiantado manifestó el grado de satisfacción con los resultados obtenidos durante el periodo de enseñanza remota. El 49.2\% confirmó sentirse sumamente satisfecho, el $27.5 \%$ medianamente y sólo un $23.3 \%$ se sintió insatisfecho con los resultados. Finalmente, se les pidió al estudiantado que evaluara, bajo una escala ordinal (de "malo" a "bueno"), algunos aspectos relacionados con la estrategia de enseñanza remota. La tabla 5 muestra tales resultados:

Tabla 5.

Evaluación del apoyo recibido por parte del estudiantado por nivel de estudios

\begin{tabular}{|l|l|l|l|l|l|l|}
\hline \multirow{2}{*}{ Elemento evaluado } & \multicolumn{3}{|c|}{ Bachillerato } & \multicolumn{3}{c|}{ Licenciatura } \\
\cline { 2 - 7 } & Malo & Regular & Bueno & Malo & Regular & Bueno \\
\hline $\begin{array}{l}\text { Los materiales y recursos gestiona- } \\
\text { dos por el profesorado y la institu- } \\
\text { ción educativa para apoyar las cla- } \\
\text { ses a distancia }\end{array}$ & $60.0 \%$ & $28.0 \%$ & $12.0 \%$ & $51.6 \%$ & $25.3 \%$ & $23.1 \%$ \\
\hline $\begin{array}{l}\text { El uso que ha hecho el profesorado } \\
\text { de la tecnología educativa a su al- } \\
\text { cance }\end{array}$ & $60.0 \%$ & $24.0 \%$ & $16.0 \%$ & $37.4 \%$ & $33.0 \%$ & $29.6 \%$ \\
\hline $\begin{array}{l}\text { Los medios y canales utilizados por } \\
\text { el profesorado para aclarar dudas y } \\
\text { explicar contenidos complejos }\end{array}$ & $64.0 \%$ & $28.0 \%$ & $8.0 \%$ & $44.0 \%$ & $33.0 \%$ & $23.0 \%$ \\
\hline $\begin{array}{l}\text { La cantidad de actividades y tareas } \\
\text { escolares solicitadas por el profeso- } \\
\text { rado durante la emergencia sanitaria }\end{array}$ & $60.0 \%$ & $28.0 \%$ & $12.0 \%$ & $57.1 \%$ & $24.2 \%$ & $18.7 \%$ \\
\hline $\begin{array}{l}\text { La forma en que el profesorado eva- } \\
\text { luó las actividades escolares desa- } \\
\text { rrolladas durante la pandemia. }\end{array}$ & $40.0 \%$ & $36.0 \%$ & $24.0 \%$ & $49.5 \%$ & $23.0 \%$ & $27.5 \%$ \\
\hline
\end{tabular}

Nota: Elaboración propia con base en los resultados del SPSS V. 21

Es posible observar que la evaluación realizada por el estudiantado difiere en algunos aspectos. Iniciando por el alumnado de bachillerato, la calificación más frecuente en todos los casos es negativa, con frecuencias que superan el $60 \%$ de la distribución en casi todos los elementos evaluados. Para ellos, los medios y canales utilizados por los/las docentes para aclarar dudas y explicar contenidos complejos sería el aspecto ejercido de peor manera, mientras que la forma de evaluación sería la menos penalizada. El alumnado de licenciatura por su parte, también fue bastante severo en su evaluación, ya que al igual que sus pares de bachillerato, puntuaron en mayor medida de forma negativa, aunque con proporciones ligeramente menores. En su caso, la cantidad de actividades y tareas escolares solicitadas por el profesorado durante la emergencia sanitaria representa el aspecto peor evaluado, indicando un exceso de solicitudes y 
una posible saturación, mientras que el uso que ha hecho el profesorado de la tecnología educativa sería el elemento más aplaudido.

\section{Transformación y proyección de los aprendizajes}

Esta última categoría pretende hacer una reflexión sobre la forma en que se ha transformado el rol del profesorado y de cómo cambiará su forma de ejercer la docencia a partir de la experiencia obtenida durante la emergencia sanitaria. Particularmente, el $68.2 \%$ considera que ha habido una transformación profunda en el rol que venía desempeñado previo a la contingencia y el $77.3 \%$ afirma que la práctica cambiará de forma permanente a partir del cambio forzado en la modalidad de estudio. Incluso, para mejorar sus competencias en el uso de la tecnología, el profesorado ha optado por la asistencia a cursos y talleres virtuales, la consulta a otros profesionales y colegas y la revisión de tutoriales y recursos de internet.

En el caso del estudiantado, al preguntarle sobre cómo se ha transformado su a partir de la experiencia obtenida durante la emergencia sanitaria, han manifestado sentir nostalgia por algunos aspectos del modelo presencial, específicamente la posibilidad de interactuar con el profesorado (50.9\%), la forma en que se desarrollaban las clases $(34.5 \%)$ y la convivencia con otros compañeros $(11.2 \%)$.

Por último, al preguntar sobre los aprendizajes que la experiencia de enseñanza remota les había dejado, tanto al profesorado como al alumnado, se destacaron múltiples reflexiones relevantes. El profesorado reflexionó sobre la poca preparación que las instituciones, el estudiantado, las familias y los propios profesores tenían para enfrentar la situación, la importancia del manejo de las tecnologías de la información y la comunicación para poder continuar en la modalidad a distancia, la necesidad de capacitación de todos los actores en diversos aspectos del proceso de enseñanza aprendizaje y la obligación de ser sensibles a las diferencias entre los estudiantes y sus desigualdades. Por otro lado, el estudiantado se enfocó en algunos valores y actitudes que tuvieron que desarrollar con el cambio de modalidad, como paciencia, autonomía, responsabilidad, organización (de tiempos y tareas), resiliencia y manejo del estrés y la presión. Resaltaron, al igual que el profesorado, la importancia del manejo de la tecnología y las carencias que alumnos y profesores tienen sobre diversos aspectos del proceso de enseñanza aprendizaje. Finalmente, muchos aprovecharon el espacio para reconocer la labor del profesorado frente a las dificultades y la necesidad de valorar su esfuerzo.

\section{Discusión}

Los hallazgos indican que tanto para el profesorado al igual que para el estudiantado, el equipo más utilizado durante la enseñanza remota de emergencia fue la computadora o laptop, lo que se explica considerando las ventajas que brinda este dispositivo para atender a las distintas tareas escolares y docentes. Asimismo, se observó la utilización de otros dispositivos, como el teléfono inteligente, en calidad de recurso didáctico de importancia, corroborando la gran penetración que ha conseguido este medio de comunicación en los distintos estratos sociales debido a su ubicuidad, facilidad de uso, personalización y a su cada vez más diversas funciones que rebasan a las de comunicación e involucran otras de tipo multimedia y ofimáticas (Pascuas-Rengifo et al., 2020; Mendoza, 2014). En sentido general se recurrió a la implementación de estrategias multicanal que atendieron a las condiciones y recursos existentes y a las distintas finalidades de las actividades en cuestión: comunicación, búsqueda de información, trabajo académico, almacenamiento, envío y recepción de tareas, entre otras. En este mismo ámbito de análisis, la comunicación entre los actores fue considerada como adecuada, siendo los grupos de WhatsApp, el recurso más popular para la interacción entre docentes y estudiantes, coincidiendo con otras investigaciones (Bocchio, 2020).

En relación con las acciones institucionales emprendidas para garantizar la continuidad de las clases a distancia, el estudiantado señaló como las principales el envío de actividades escolares por correo electrónico u otro medio y la habilitación de plataformas para continuar las 
clases en línea. Mientras que, al referirse a los recursos utilizados por el personal docente, destacaron el uso de libros y materiales digitalizados y el uso de videos (Lederman, 2020). En cuanto a conectividad, sólo una cuarta parte del estudiantado señaló contar con servicio de internet de banda ancha, aunado a quienes confirmaron no tener ninguna forma de conexión a internet en el hogar. Datos que revelan disparidades en el acceso a los recursos y dispositivos tecnológicos (Luthra, 2020). En el caso del profesorado, indicaron disponer de internet de banda ancha, o bien, acceso desde datos móviles garantizando la conexión a internet.

En este sentido, los resultados invitan a considerar la existencia de una doble brecha. Las limitantes enunciadas en términos de infraestructura tecnológica y el incipiente desarrollo de habilidades y competencias digitales necesarias para un uso efectivo de tales recursos, propios de los entornos virtuales (Almazán, 2020; Luthra, 2020; Pardo \& Cobo, 2020; Ruíz, 2020). Por lo que se reconoce la existencia de una brecha digital y educativa (Lloyd, 2020). La exploración ha permitido descubrir que en Educación Superior sólo una cuarta parte del alumnado tuvo una experiencia previa en educación a distancia, mientras que en Educación Media Superior la experiencia previa resulta prácticamente inexistente. La situación resulta diferente en el caso del profesorado pues manifestaron contar con mayor experiencia, aunque no todos reconocieron estar preparados para el cambio de modalidad (Menéndez \& Figares, 2020). Ello explica que, entre las principales acciones institucionales emprendidas ante la abrupta transición de la modalidad presencial a la modalidad de enseñanza remota, destaquen aquellas dirigidas a atender las necesidades de capacitación del profesorado en el uso de tecnología educativa, a través de cursos y asesorías.

De manera agregada, se ha encontrado que el contexto de emergencia sanitaria ha provocado que gran parte del profesorado aprenda sobre la marcha, improvisando y la vez, desarrollando propuestas creativas e innovadoras, que demuestran la capacidad de adaptación y flexibilidad ante los cambios. Lo anterior, ha generado un notable aumento de tiempo destinado a la preparación de clases y materiales por parte del profesorado, sumado a otras tareas docentes. Lo paradójico, resulta que un poco más del $10 \%$ del profesorado vio afectada su remuneración salarial, a pesar de encontrarse realizando un mayor esfuerzo laboral, en un escenario de mayor estrés y presión generado por la pandemia.

Con relación a las dificultades identificadas ante el cambio de modalidad, el profesorado señaló en mayor medida aquellas vinculadas con la recepción y evaluación de las actividades escolares de sus estudiantes, así como la explicación de contenidos y aclaración de dudas por medio de los canales disponibles. Aspectos relacionados con los principales obstáculos identificados en el estudiantado para cumplir con sus actividades escolares a distancia, entre los que destacan la falta de recursos tecnológicos (Disponibilidad de equipos y conexión a internet en el hogar), el poco compromiso y responsabilidad con las tareas escolares y la aparición de otras problemáticas familiares a partir de la pandemia.

Referente al apoyo recibido por parte de la institución educativa, el profesorado manifestó satisfacción con los resultados obtenidos evaluando de bueno a excelente el apoyo recibido. Por su parte el alumnado, aunque manifestó su satisfacción con los resultados obtenidos, se mostró mucho más crítico al evaluar distintos elementos de la enseñanza remota de emergencia, los cuales recibieron una calificación predominantemente negativa. En este orden de ideas, el aspecto peor evaluado en el caso de los bachilleres se relacionó con los medios y canales utilizados por el profesorado para aclarar dudas y explicar contenidos complejos. Mismo que también el profesorado reconoció entre los de mayor dificultad ante el cambio de modalidad, lo que pudiera atribuirse, a la inexperiencia y a la poca preparación en el manejo de los entornos digitales por parte de ambos actores, como ya se ha señalado (Álvarez et al., 2020). En el caso del estudiantado de licenciatura, el aspecto peor evaluado resultó la cantidad de actividades y tareas escolares solicitadas por el profesorado durante la emergencia sanitaria. Lo que ha sido un elemento característico en el tránsito abrupto de modalidad, al tratar de "transformar las clases presenciales a modo virtual, pero sin cambiar el currículum ni la metodología" (UNESCO, 2020b, p. 26). 
En cuanto a los aspectos mejor calificados, el estudiantado de bachillerato se remite a la forma en que el profesorado evaluó las actividades escolares. Cuestión comprensible, dadas las circunstancias de crisis, que invitaron a ser más sensibles ante las dificultades del estudiantado y con ello a centrar la atención en los aprendizajes, en las experiencias y no en el control y la evaluación (Hodges et al., 2020). De ahí que se convocara al profesorado a optar por alternativas que ayudaran a evitar la reprobación, como corresponde a un modelo de enseñanza remota aún no adaptado, que hace énfasis en la tolerancia y propone un menor nivel de exigencia en términos de evaluación (Pardo y Cobo, 2020). Mientras que el elemento mejor evaluado por el estudiantado de nivel licenciatura, resultó ser el uso que ha hecho el profesorado de la tecnología educativa a su alcance. Lo que pudiera relacionarse con una mayor experiencia previa en el modelo de educación a distancia, en el caso de los docentes universitarios.

Finalmente, en cuanto a los aprendizajes más relevantes, tanto docentes como estudiantes, reflexionaron en torno a la importancia de las tecnologías de la información y la comunicación, como medios indispensables para enfrentar la actual emergencia sanitaria y garantizar la continuidad educativa. A la vez reconocieron sus propias carencias y dificultades, y con ello la necesidad de fortalecer los mecanismos institucionales para apoyar a los actores escolares ante situaciones de este tipo, con espacios y recursos que ayuden a la constante capacitación y actualización en temas vinculados al uso y manejo de tecnología con fines educativos y ambientes virtuales de aprendizaje. El profesorado subrayó la necesidad de atender a las profundas desigualdades que existen en la sociedad mexicana y reconoció haber experimentado una transformación significativa en su rol como docente. Por su parte, el estudiantado hizo referencia en sus respuestas a valores y actitudes que tuvieron que desarrollar ante el cambio de modalidad, entre ellos, autonomía, responsabilidad, paciencia, una mejor administración de los tiempos para cumplir con las tareas y obligaciones escolares, entre otros aspectos. En sentido general hubo un reconocimiento explícito del esfuerzo y la dedicación mostrada por el profesorado al hacer frente a la compleja crisis ocasionada por la contingencia.

\section{Limitaciones del estudio}

Es preciso señalar que, si bien se logró tener un panorama general sobre la experiencia de docentes y alumnos en el contexto de la educación media y superior (dado el carácter exploratorio del estudio), la cantidad muestral no permitió realizar contrastes estadísticos inferenciales que pudieran contrastar de mejor manera la experiencia de docentes y estudiantes de ambos niveles educativos más allá de la descripción. Ante tal situación, se recomienda que en estudios posteriores se profundice en mayor medida sobre cada uno de los actores y niveles educativos, así como en el análisis de otros factores que pudieran estar vinculados a dicha experiencia, como el tipo de institución o el nivel socioeconómico de las familias. Incluso, mediante análisis estadísticos más sofisticados o estrategias de corte cualitativo como entrevistas a profundidad o grupos focales.

\section{Referencias}

Almazán, A. (2020). Covid-19: ¿Punto Sin Retorno de la Digitalización de la Educación? Revista Internacional De Educación Para La Justicia Social, 9(3), 1-4. Recuperado de https://revistas.uam.es/riejs/article/view/12089

Álvarez, M., Gardyn, N., Iardelevsky, A., \& Rebello, G. (2020). Segregación Educativa en Tiempos de Pandemia: Balance de las Acciones Iniciales durante el Aislamiento Social por el Covid-19 en Argentina. Revista Internacional De Educación Para La Justicia Social, 9(3), 25-43. Recuperado de https://revistas.uam.es/riejs/article/view/12268

Arce-Peralta, F. (2020). La transición del paradigma educativo hacia nuevos escenarios: COVID-19. CienciAmérica, 9(2), 115-119. doi: http://dx.doi.org/10.33210/ca.v9i2.285

Arriagada, P. (2020). Pandemia Covid-19: Educación a Distancia. O las Distancias en la Educación. Revista Internacional De Educación Para La Justicia Social, 9(3), 1-3. Recuperado de https://revistas.uam.es/riejs/article/view/12396 
Bocchio, M. (2020). El Futuro Llegó Hace Rato: Pandemia y Escolaridad Remota en Sectores Populares de Córdoba, Argentina. Revista Internacional De Educación Para La Justicia Social, 9(3), 1-10. Recuperado de https://revistas.uam.es/riejs/article/view/12473

Bonilla-Guachamín, J. (2020). Las dos caras de la educación en el COVID-19. CienciAmérica, 9(2), 89-98. doi: http://dx.doi.org/10.33210/ca.v9i2.294

Bustamante, R. (2020) Educación en cuarentena: cuando la emergencia se vuelve permanente. Aportes para el Diálogo y la Acción, 1(1), 1-9. Recuperado de http://www.grade.org.pe/creer/archivos/Art\%C3\%ADculo-Roberto-Bustamante-parte1.pdf

Cáceres-Piñaloza, K. (2020). Educación virtual: Creando espacios afectivos, de convivencia y aprendizaje en tiempos de COVID-19. CienciAmérica, 9(2), 38-44. doi: http://dx.doi.org/10.33210/ca.v9i2.284

Casal, L. \& Fernández, J. (2020). La Respuesta Jurídica ante el Covid-19 y su Incidencia en la Educación. Revista Internacional De Educación Para La Justicia Social, 9(3), 1-7. Recuperado de https://revistas.uam.es/riejs/article/view/12436

Cervantes, E. \& Gutiérrez, P. (2020). Resistir la Covid-19. Intersecciones en la Educación de Ciudad Juárez, México. Revista Internacional De Educación Para La Justicia Social, 9(3). Recuperado de https://revistas.uam.es/riejs/article/view/12096

Cifuentes-Faura, J. (2020). Consecuencias en los Niños del Cierre de Escuelas por Covid-19: El Papel del Gobierno, Profesores y Padres. Revista Internacional De Educación Para La $\begin{array}{lllll}\text { Justicia } & \text { Social, } & \text { 9(3), } & \text { Recuperado }\end{array}$ https://revistas.uam.es/riejs/article/view/12216

Cóndor-Herrera, O. (2020). Educar en tiempos de COVID-19. CienciAmérica, 9(2), 31-37. doi: https://dx.doi.org/10.33210/ca.v9i2.281

García-García, M. (2020). La docencia desde el hogar. Una alternativa necesaria en tiempos del Covid 19. Polo del Conocimiento, 5(4), 304-324. doi: http://dx.doi.org/10.23857/pc.v5i4.1386

Hernández, R. \& Mendoza, C. (2018). Metodología de la investigación: Las rutas cuantitativa, cualitativa y mixta. México: McGraw Hill.

Hodges, C., Moore, S., Lockee, B., Trust, T., \& Bond, A. (2020). The Difference Between Emergency Remote Teaching and Online Learning. Educause. Recuperado de https://er.educause.edu/articles/2020/3/the-difference-between-emergency-remoteteaching-and-online-learning

Jiménez, J. (2020). Polémicas Educativas en Confinamiento. Revista Internacional De Educación Para La Justicia Social, 9(3), 1-5. Recuperado de https://revistas.uam.es/riejs/article/view/12084

Lederman, D. (2020) Will Shift to Remote Teaching Be Boon or Bane for Online Learning? Inside Higher. Recuperado de https://www.insidehighered.com/digitallearning/article/2020/03/18/most-teaching-going-remote-will-help-or-hurt-onlinelearning

Lloyd, M. (2020) Desigualdades educativas y la brecha digital en tiempos de Covid-19 en Educación y pandemia. Una visión académica Instituto de Investigaciones sobre la Universidad y la Educación. México, UNAM. Recuperado de http://www.iisue.unam.mx/nosotros/covid/educacion-y-pandemia

Luthra, P. (2020) Una ocasión para reinventar la escuela. Correo de la UNESCO: un solo mundo, voces múltiples. Recuperado de https://es.unesco.org/courier/2020-3/ocasionreinventar-escuela

Mendoza, M. (2014). El teléfono celular como mediador en el proceso de enseñanzaaprendizaje. Omnia, 20(3), 9-22. Recuperado de https://www.redalyc.org/articulo.oa?id=737/73737091002

Menéndez, D. \& Figares, J. (2020). Retos Educativos durante el Confinamiento: La Experiencia con Alumnos con Necesidades Educativas Especiales. Revista Internacional De Educación Para La Justicia Social, 9(3), 1-11. Recuperado de https://revistas.uam.es/riejs/article/view/12229 
Mérida, Y. \& Acuña, L. (2020). Covid-19, Pobreza y Educación en Chiapas: Análisis a los Programas Educativos Emergentes. Revista Internacional De Educación Para La Justicia Social, 9(3), 61-82. Recuperado de https://revistas.uam.es/riejs/article/view/12435

Morales, J. (2020). Oportunidad o Crisis Educativa: Reflexiones desde la Psicología para Enfrentar los Procesos de Enseñanza-Aprendizaje en Tiempos de Covid-19. Revista Internacional De Educación Para La Justicia Social, 9(3), 1-9. Recuperado de https://revistas.uam.es/riejs/article/view/12228

Moreno-Rodríguez, R. (2020). Reflexiones en torno al Impacto del Covid-19 sobre la Educación Universitaria: Aspectos a Considerar acerca de los Estudiantes con Discapacidad. Revista Internacional De Educación Para La Justicia Social, 9(3). 1-6. Recuperado de https://revistas.uam.es/riejs/article/view/12227

Muñoz, J. \& Lluch, L. (2020). Educación y Covid-19: Colaboración de las Familias y Tareas Escolares. Revista Internacional De Educación Para La Justicia Social, 9(3) 1-17. https://revistas.uam.es/riejs/article/view/12182

Pardo. H. y Cobo, C. (2020). Expandir la universidad más allá de la enseñanza remota de emergencia Ideas hacia un modelo híbrido post-pandemia. Outliers School. Barcelona

Pascuas-Rengifo, Y., García-Quintero, J., \& Mercado-Varela, M. (2020). Dispositivos móviles en la educación: tendencias e impacto para la innovación. Revista Politécnica, 16(31), 97-109. doi: https://doi.org/10.33571/rpolitec.v16n31a8

Pérez-Narváez, M. \& Tufiño, A. (2020). Teleeducación y COVID-19. CienciAmérica, 9(2), 5864. doi: http://dx.doi.org/10.33210/ca.v9i2.296

Quintana, I. (2020). Covid-19 y Cierre de Universidades ¿Preparados para una Educación a Distancia de Calidad? Revista Internacional De Educación Para La Justicia Social, 9(3), 111. Recuperado de https://revistas.uam.es/riejs/article/view/12232

Rodríguez del Rincón, P. (2020). Alumnos de Necesidades Educativas Especiales, en Casa por el Covid-19. Experiencias que nos Descubren Vidas. Revista Internacional De Educación Para La Justicia Social, 9(3), 1-13. Recuperado de https://revistas.uam.es/riejs/article/view/12092

Ruiz, G. (2020). Marcas de la Pandemia: El Derecho a la Educación Afectado. Revista Internacional De Educación Para La Justicia Social, 9(3), 45-59. Recuperado de https://revistas.uam.es/riejs/article/view/12355

Suárez, N. (2020). Formación docente universitaria y crisis sanitaria COVID-19. CienciAmérica, 9(2), 109-114. doi: http://dx.doi.org/10.33210/ca.v9i2.299

UNESCO (2020a) Aportes para una respuesta educativa frente al COVID-19 en América Latina. Oficina Regional de Educación para América Latina y el Caribe (OREALC/UNESCO Santiago). Recuperado de https://unesdoc.unesco.org/ark:/48223/pf0000373761

UNESCO (2020b) Covid-19 y educación superior: de los efectos inmediatos al día después. Análisis de impactos, respuestas políticas y recomendaciones. Instituto Internacional de la UNESCO para la Educación Superior en América Latina y el Caribe (IESALC). Recuperado de http://www.iesalc.unesco.org/wp-content/uploads/2020/05/COVID-19-ES130520.pdf

Varela, C., Lorenzo, M. \& García-Álvarez, J. (2020). La Escuela en Prisión ante el Covid-19. Un Desafío Sobre el que Repensar la Educación. Revista Internacional De Educación Para La Justicia Social, 9(3), 1-12. Recuperado de https://revistas.uam.es/riejs/article/view/12469

Vivanco-Saraguro, A. (2020). Teleducación en tiempos de COVID-19: brechas de desigualdad. CienciAmérica, 9(2), 166-175. doi: http://dx.doi.org/10.33210/ca.v9i2.307 